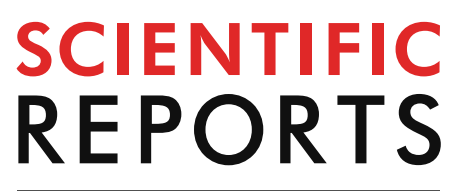

natureresearch

Check for updates

\title{
Standardized distances
}

\section{for placement of REBOA in patients with aortic stenosis}

\author{
Markus Harboe Olsen ${ }^{1 \bowtie}$, Tasalak Thonghong ${ }^{2}$, Lars Søndergaard ${ }^{2}$ \& Kirsten Møller $^{1}$
}

Resuscitative endovascular balloon occlusion of the aorta (REBOA) is a technique where a balloon is advanced through the common femoral artery and temporarily inflated for treatment of cardiac arrest or non-compressible haemorrhage. The aim of this study was to measure intravascular distances relevant for correct placement of the REBOA catheter using computer tomographic (CT) scans. In a series of CT scans of the aorta from 100 patients diagnosed with severe aortic stenosis planned for transcatheter aortic valve implantation, we measured the intravascular distance from the insertion site in the common femoral artery to two potential zones for placement of the REBOA catheter; between the left subclavian artery and the celiac trunk (Zone 1), as well as between the aortic bifurcation and the distal take-off of the renal arteries (Zone 3). The mean ( $\pm S D$ ) intravascular distance from the femoral artery to intra-aortic Zone 1 was $36( \pm 2.5) \mathrm{cm}$ for the lower border and $60( \pm 4.1) \mathrm{cm}$ for the upper border, respectively. For intra-aortic Zone 3 , the mean $( \pm S D)$ intravascular distance was $21( \pm 2.1) \mathrm{cm}$ to the lower border and $31( \pm 2.3) \mathrm{cm}$ to the upper border. Calculated potentially safe intervals for placement of the REBOA in Zone 1 was with $99.7 \%$ likelihood between 43 and $48 \mathrm{~cm}$. No similar potentially safe interval could be calculated for Zone 3. According to this cohort study of patients with severe aortic stenosis, the balloon of the REBOA catheter should travel intraarterially between 43 (lower limit) and $48 \mathrm{~cm}$ (upper limit) from the site of insertion into the common femoral artery, which would lead to correct placement in intra-aortic Zone 1 in $99.7 \%$ of cases. In contrast, no potential safety interval could be similarly defined for insertion in Zone 3.

Until the introduction of resuscitative endovascular balloon occlusion of the aorta (REBOA), compression of the descending or abdominal aorta was done with external clamping via resuscitative thoracotomy or laparotomy during severe haemorrhage to minimize blood loss ${ }^{1,2}$. Occlusion of the aorta minimizes the haemorrhage from arteries distal to the occlusion and simultaneously redirects the circulating blood to upper body, e.g. the coronary and cerebral vascular territories ${ }^{3}$. Complete occlusion of the aortic lumen is possible for several minutes without complications, which may allow for surgical repair of the injur $y^{4-6}$, or for cardio-pulmonary resuscitation to achieve recovery of spontaneous circulation in the case of cardiac arrest.

Depending on the type of injury, the REBOA catheter is inserted intra-arterially through the common femoral artery and a balloon is inflated when it is located between the celiac trunk and the left subclavian artery (Zone 1 ), or between the aortic bifurcation and the distal border of the renal arteries (Zone 3). In contrast, occlusion between the celiac trunk and the distal border of the renal artery (Zone 2) is generally not recommended (the so-called no-occlusion zone) (Fig. 1) ${ }^{7}$. The indications for REBOA include, but are not limited to, intraabdominal and pelvic hemorrhage, as well as traumatic and non-traumatic cardiac arrest ${ }^{8}$.

Inflation with the balloon located in Zone 1 is used for resuscitation after non-compressible torso hemorrhage or after cardiac arrest. In this case, the length of the REBOA catheter should allow balloon placement above the celiac trunk to stop perfusion of the lower trunk and extremities but must be placed below the lower limit of the left subclavian artery to protect cerebral perfusion. Conversely, Zone 3 occlusion is used for uncontrollable pelvic and lower-extremity haemorrhage, which cannot be controlled by tourniquets; for this indication, the balloon should be located above the aortic bifurcation and below the lower renal artery.

${ }^{1}$ Department of Neuroanaesthesiology, Rigshospitalet, University of Copenhagen, Copenhagen, Denmark. ${ }^{2}$ Department of Cardiology, Rigshospitalet, University of Copenhagen, Copenhagen, Denmark. ${ }^{\square}$ email: oel@oelfam.com 


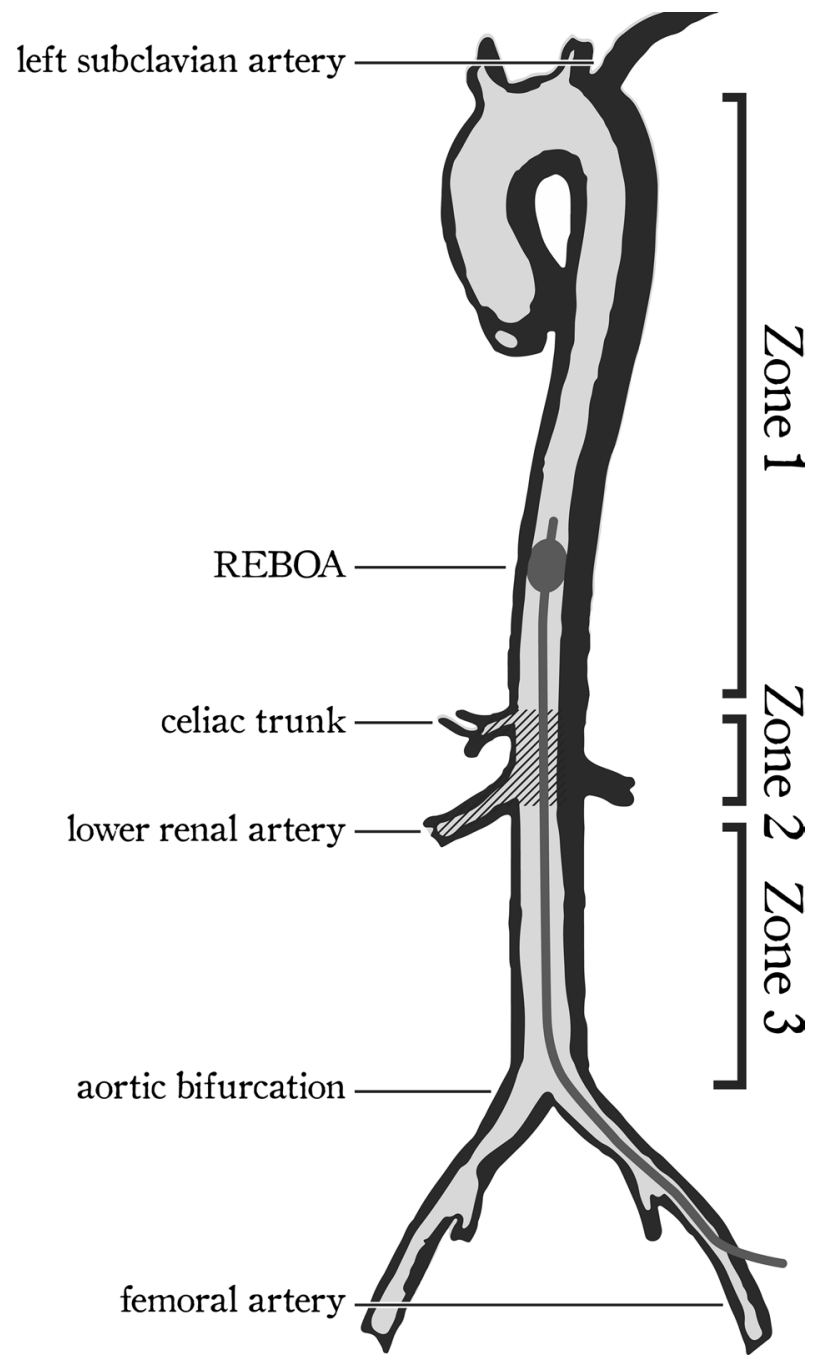

Figure 1. Simplistic rendering of aorta. Zone 1 (from left subclavian artery to the upper border of the celiac trunk), Zone 2 (the upper border of the celiac trunk to the lower border of the distal take-off of the renal arteries), and Zone 3 (from the lower border of the lower renal artery to the aortic bifurcation). Zone 1 is occluded in the case of cardiac arrest or life-threatening intra-abdominal hemorrhage; Zone 2 has no current indication; and Zone 3 is occluded in the case of life-threatening pelvic or lower limb haemorrhage ${ }^{7}$. REBOA Resuscitative Endovascular Balloon Occlusion of the Aorta.

A major current limitation in the use of the REBOA device is the need of radiographic guidance for safe placement, which considerably limits its prehospital or bedside usage ${ }^{2}$. The available literature on REBOA catheter placement without radiographic guidance ${ }^{9-12}$ includes both successes and failures; thus an effective non-radiography-based REBOA method has yet to be developed ${ }^{13-15}$. In the meantime, unguided placement of REBOA carries a risk of incorrect positioning of the occluding balloon, potentially leading to balloon rupture, pressure damage, non-perfusion of critical organs or unintended perfusion of non-critical organs or hemorrhaging arteries. Length markers and external measurement of the patient torso have both been investigated as methods to determine zone placement of the REBOA catheter. However, further simplification of the setup may conceivably increase the success rate, decrease the time to catheter insertion and successful balloon inflation ${ }^{14,15}$ and optimize the feasibility of REBOA treatment in the prehospital or bedside setting.

One such simplification is to calculate a standardized insertion length of the REBOA catheter that leads to correct placement of the balloon in the desired intra-aortic zone with an acceptable success rate. The first step would be to investigate the intravascular distances from the hypothetical insertion point to anatomical landmarks that define the borders of these zones in a relevant patient population.

The aim of this study was to test the hypothesis that standardized insertion lengths of a REBOA catheter balloon may ensure inflation in intra-aortic Zone 1 or 3, respectively, without radiographic guidance and irrespective of the height of individual patients. To this end, we measured intravascular distances from a well-defined entry point in the left common femoral artery to anatomical landmarks and calculated confidence intervals for the distances that would ensure correct location inside intra-aortic Zones 1 and 3, respectively. Previously only 


\begin{tabular}{|c|c|c|c|c|c|c|c|c|c|c|}
\hline & \multicolumn{3}{|c|}{ All $(n=100)$} & \multicolumn{3}{|c|}{ Men $(n=62)$} & \multicolumn{3}{|c|}{ Women $(n=38)$} & \multirow[b]{2}{*}{$P$ value ${ }^{\star}$} \\
\hline & Mean & SD & Range & Mean & SD & Range & Mean & SD & Range & \\
\hline Age (years) & 78 & 8 & $42 ; 89$ & 76 & 9 & $42 ; 88$ & 78 & 6 & $66 ; 89$ & $<0.05$ \\
\hline Height $(\mathrm{cm})$ & 170 & 10 & $140 ; 193$ & 175 & 7 & $162 ; 193$ & 162 & 8 & $140 ; 178$ & $<0.0001$ \\
\hline BMI $\left(\mathrm{kg} / \mathrm{m}^{2}\right)$ & 26.5 & 5.1 & $17.0 ; 44.7$ & 27.4 & 5.3 & $19.1 ; 44.7$ & 25.0 & 4.42 & $17 ; 42$ & $<0.05$ \\
\hline
\end{tabular}

Table 1. Characteristics of the study population. SD standard deviation, BMI body mass index. ${ }^{\star}$ Student’s $t$ test for unpaired samples, men versus women.

trauma patients have been studied ${ }^{16,17}$, and with the recent proposal to include of REBOA in treatment of nontraumatic cardiac arrest this study is done in an elderly population.

\section{Materials and methods}

A series of computer tomographic scans (CT) from randomly selected patients who had previously been diagnosed with severe, symptomatic aortic stenosis and planned for transcatheter aortic valve implantation (TAVI) were retrospectively evaluated. All scans had been conducted between June 2017 and April 2018 and were selected from the archive at the Department of Cardiology, Rigshospitalet, Denmark (Table 1).

The insertion site for the balloon catheter was defined as a point in the left femoral artery $3 \mathrm{~cm}$ below the mid-inguinal point, i.e. the midpoint between the anterior superior iliac spine and pubic tubercle. Aortic zones were defined as follows: Zone 1: the zone extending from the upper border of the celiac trunk to the lower border of the left subclavian artery; Zone 2, from the lower border of the lowest of the renal arteries to the upper border of the celiac trunk; and Zone 3, from the aortic bifurcation to the lower border of the lowest of the renal arteries (Fig. 1)

The intravascular distance from the baseline to the following anatomical landmarks was measured: the aortic bifurcation; the lower border of the lowest of the renal arteries; the lower border of the celiac trunk; the center of the celiac trunk; the upper border of the celiac trunk; the level of the diaphragm; and the lower border of the left subclavian artery. All distances were measured, by one clinician (TT), in the midline of the femoral, external and common iliac arteries and the aorta. All measurements were made in 3MENSIO (Maastricht, The Netherlands, 2018) by a specialist cardiologist at the Department of Cardiology, Rigshospitalet, Denmark.

With the use of available scans and data the Regional Committee in the Capital Region of Denmark on Health Research Ethics (Protocol number: 17024428; September 8, 2017) ruled that according to Danish law no approval or consent from patients were necessary. The study was furthermore approved by the Director of the Department of Cardiology, Rigshospitalet, Denmark, and carried out in accordance with regional guidelines.

Statistical analysis. Analyses were performed using R (R 4.0.0, R Core Team [2020], Vienna, Austria) ${ }^{18}$. All data were normally distributed as asserted by histograms and the Shapiro-Wilk test. Quantitative data were given as mean $(\mathrm{SD})$.

Continuous variables were compared between groups using Student's $t$ test for unpaired data. For measurement of anatomical distances, the mean as well as 95\% (2 SD) and 99.7\% (3 SD) confidence limits were calculated for potentially safe placement of a REBOA catheter balloon inside intra-aortic zones. This allowed us to determine the $95 \%$ and $99.7 \%$ intervals of potentially safe placement of the REBOA balloon in Zone 1 and 3 as follows:

In Zone 1, the potentially safe interval was located between the upper confidence limit of the distance to the upper border of the celiac trunk (the lower limit of Zone 1) and the lower confidence limit of the distance to the lower border of the subclavian artery (the upper limit);

In Zone 3, the potentially safety interval was located between the upper confidence limit of the distance to the aortic bifurcation (the lower limit of the Zone 3) and the lower confidence limit of the distance to the lower border of the most distal renal artery (the upper limit).

Linear regression was used to measure an association between distances to anatomical landmarks and height, weight, age and sex. $P$ values of $<0.05$ were considered significant.

Ethics approval and consent to participate. With the use of available scans and data the Regional Committee on Health Research Ethics (Protocol number: 17024428) ruled that according to Danish law no approval was necessary. The study was furthermore approved by the Director of the Department of Cardiology, Rigshospitalet, Denmark.

\section{Results}

One hundred patients with aortic valve stenosis (62 males and 38 females) were included (Table 1). Distances from the common femoral artery to anatomical landmarks are given in Table 2 . Sex and height were significantly associated with the distances to the landmarks that defined the borders of both Zone 1 and Zone 3 (Supplemental Data A).

The $99.7 \%$ safety interval for the intra-aortic Zone 1 was 43 to $48 \mathrm{~cm}$, whereas the $95 \%$ safety interval was 41 to $52 \mathrm{~cm}$. As expected, no patients in the cohort had anatomical distances that would lead to incorrect placement of the REBOA catheter balloon, if it was placed inside the $99.7 \%$ safety interval in Zone 1 . In contrast, using the 95\% safety interval, four patients in this cohort would have experienced incorrect balloon placement (Fig. 2a). Similar subgroup analyses resulted in only a marginal change in the safety intervals between male and female (Fig. 2b,c). 


\begin{tabular}{|c|c|c|c|c|c|c|c|c|c|}
\hline & \multicolumn{3}{|c|}{ All $(n=100)$} & \multicolumn{3}{|c|}{$\operatorname{Men}(n=62)$} & \multicolumn{3}{|c|}{ Women $(n=38)$} \\
\hline & Mean & SD & Range & Mean & SD & Range & Mean & SD & Range \\
\hline B to distal to left subclavian artery & 60 & 4.1 & $50 ; 69$ & 61 & 4 & $52 ; 69$ & 58 & 3.5 & $50 ; 65$ \\
\hline B to diaphragm & 39 & 3.2 & $32 ; 49$ & 39 & 3.2 & $32 ; 49$ & 39 & 3.2 & $34 ; 45$ \\
\hline B to proximal to celiac trunk & 36 & 2.5 & $30 ; 42$ & 37 & 2.3 & $32 ; 42$ & 35 & 2.5 & $30 ; 40$ \\
\hline B to celiac trunk & 36 & 2.5 & $31 ; 44$ & 36 & 2.2 & $31 ; 41$ & 35 & 2.5 & $30 ; 40$ \\
\hline B to distal to celiac trunk & 35 & 2.5 & $29 ; 41$ & 36 & 2.2 & $31 ; 41$ & 34 & 2.5 & $29 ; 40$ \\
\hline B to distal to renal artery & 31 & 2.3 & $25 ; 37$ & 32 & 2.1 & $27 ; 37$ & 30 & 2.1 & $25 ; 35$ \\
\hline B to aortic bifurcation & 21 & 2.1 & $16 ; 28$ & 22 & 2.2 & $17 ; 28$ & 21 & 1.8 & $16 ; 25$ \\
\hline B to middle of Zone $1^{a}$ (calc.) & 48 & 3.1 & $41 ; 55$ & 49 & 2.9 & $42 ; 55$ & 47 & 2.9 & $40 ; 53$ \\
\hline B to middle of Zone $2^{\mathrm{b}}$ (calc.) & 34 & 2.3 & $28 ; 40$ & 34 & 2.1 & $29 ; 40$ & 32 & 2.1 & $28 ; 38$ \\
\hline $\mathrm{B}$ to middle of Zone $3^{\mathrm{c}}$ (calc.) & 26 & 2.0 & $21 ; 32$ & 27 & 2 & $23 ; 32$ & 25 & 1.8 & $21 ; 30$ \\
\hline
\end{tabular}

Table 2. Distance from baseline to anatomical locations $(\mathrm{cm}) . B$ baseline, $S D$ standard deviation, Calc. calculated positions. ${ }^{\mathrm{a} B e t w e e n}$ below left subclavian artery and above celiac trunk. ${ }^{\mathrm{b}}$ Between below lowest renal artery and above celiac trunk. ${ }^{c}$ Between below lowest renal artery and aortic bifurcation.

a: Zone 1 - All $(n=100)$

from above celiac trunk and below left subclavian artery

$$
\text { from above celiac trunk and below left subclavian artery }
$$

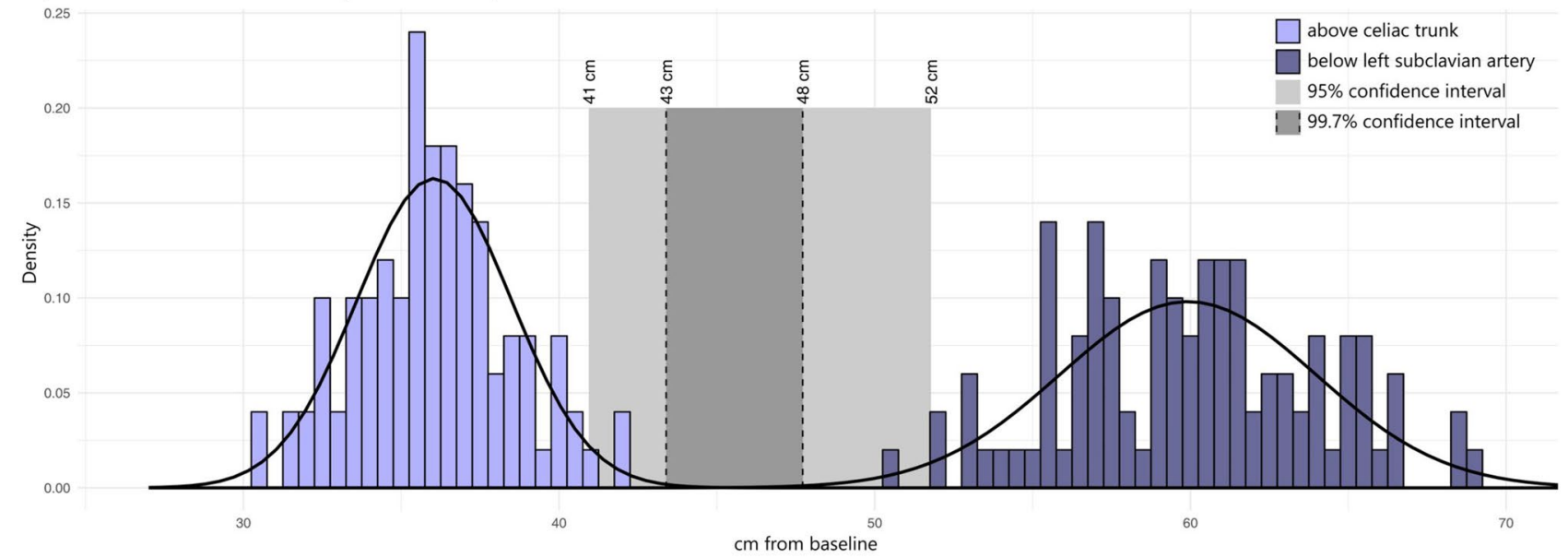

b: Zone 1 - Female ( $n=38)$

c: Zone 1 - Male $(n=62)$

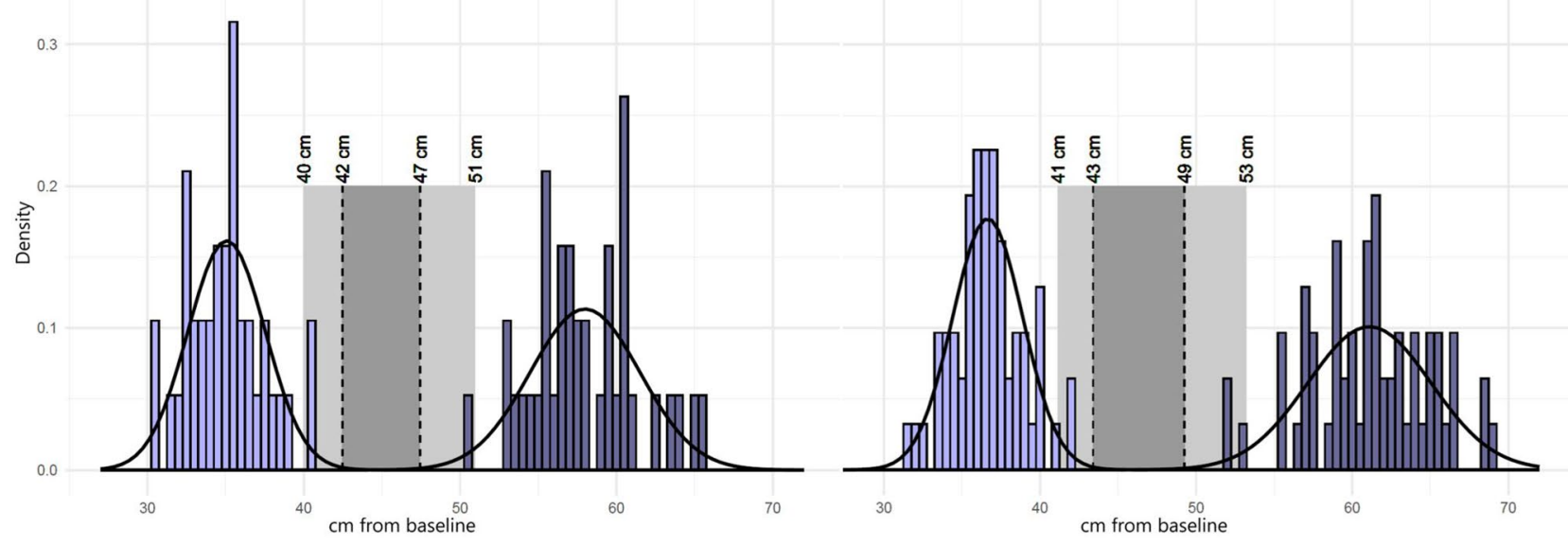

Figure 2. Location of Zone 1 in $\mathrm{cm}$ from the femoral arterial baseline in all (a), in female (b) and in male (c). The upper border (below left subclavian artery) and lower border (above celiac trunk) of Zone 1 were plotted into a histogram with grouping data in blocks of $0.5 \mathrm{~cm}$ and above a calculated normal distribution curve. Light grey rectangle defines the $95 \%$ confidence interval for placement in Zone 1 . Dark grey rectangle defines the 99.7\% confidence interval for placement in Zone 1. 
a: Zone 3 - All ( $n=100)$

from aortic bifurcation and below lower renal artery

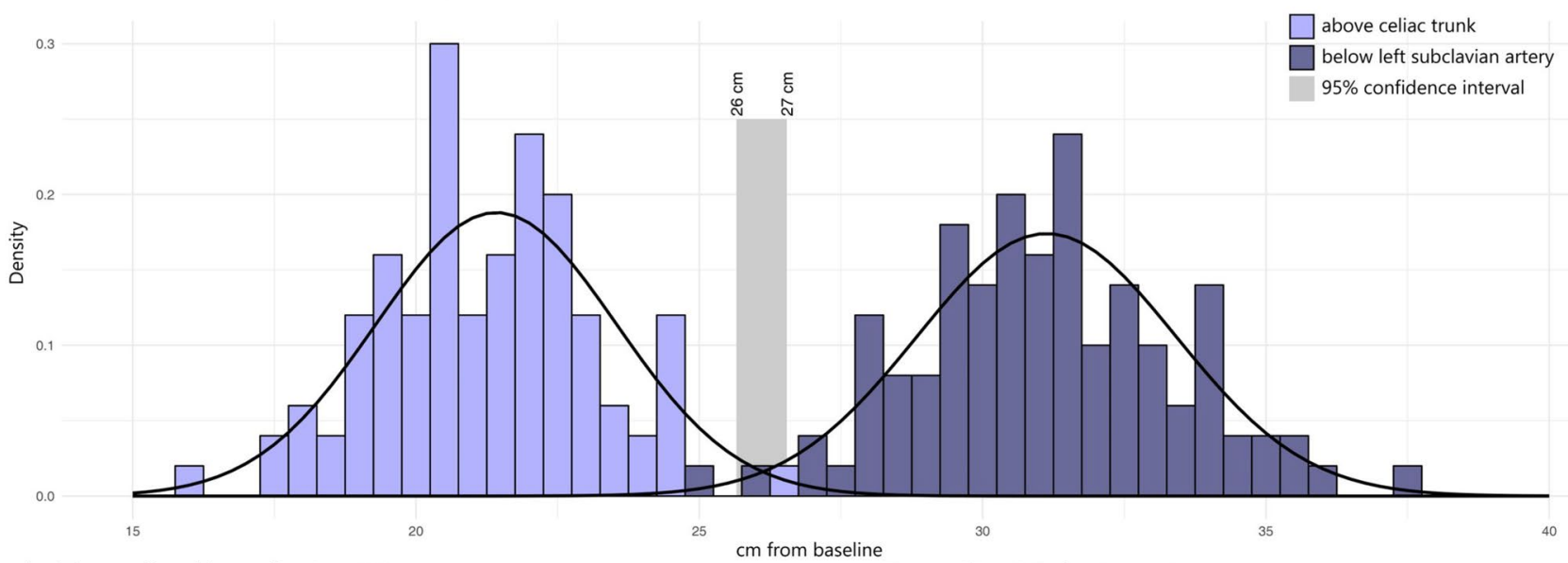

b: Zone 3 - Female $(n=38)$

c: Zone 3 - Male ( $n=62$ )

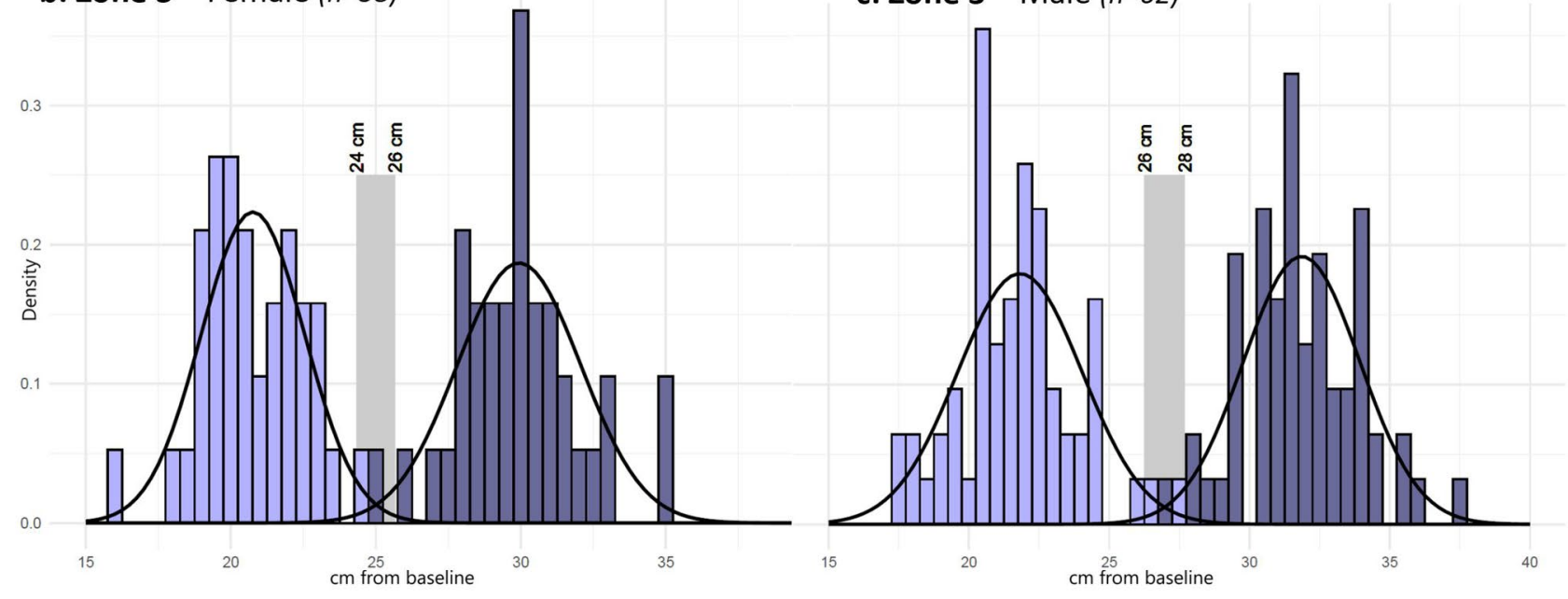

Figure 3. Location of Zone 3 in $\mathrm{cm}$ from the femoral arterial baseline in all (a), in female (b) and in male (c). The upper border (below lower renal artery) and lower border (at aortic bifurcation) of Zone 1 were plotted into a histogram with grouping data in blocks of $0.5 \mathrm{~cm}$ and above a calculated normal distribution curve. Light grey rectangle defines the $95 \%$ confidence interval for placement in Zone 3. No 99.7\% confidence interval could be defined for placement in Zone 3.

For Zone 3, the $99.7 \%$ safety interval of could not be defined, because the $99.7 \%$ confidence limits of the upper and lower border overlapped each other. However, a 95\% safety interval was calculated as 26 to $27 \mathrm{~cm}$; in the present cohort, six patients would have received incorrectly placed balloon, if this safety interval had been used (Fig. 3a). The subgroup analyses showed the $95 \%$ safety interval varied between genders (Fig. $3 \mathrm{~b}+\mathrm{c})$.

\section{Discussion}

To ensure a minimal risk of wrong placement of REBOA, a $99.7 \%$ confidence point was preferable even though a $95 \%$ confidence interval is normally acceptable. With this in mind, this study suggests a relatively safe interval for placement of the REBOA catheter balloon in Zone 1 between 43 and $48 \mathrm{~cm}$ from the hypothetical baseline in the femoral artery. In contrast, an equally safe interval could not be defined in Zone 3.

The upper limit of Zone $1(48 \mathrm{~cm})$ found in our study was somewhat higher than the limits reported in three previous publications investigating the placement of $\operatorname{REBOA}^{16,19,20}$ (Supplemental Data B), but corresponds with a large study investigating REBOA placement in a large trauma population ${ }^{17}$. This suggests that the optimal placement should be in the lower part of Zone 1, to minimize the risk of misplacement, also when adding genderrelated differences into the equation. Additionally, linear regression suggested a relationship between height and the safety limits of the zone. Thus, our findings may not apply to populations with different distributions of height.

Zone 3 is shorter than Zone 1 , and we were unable to identify a similar potentially safe interval to place the REBOA device in Zone 3. With 95\% certainty placement could be placed between 26 and $27 \mathrm{~cm}$, and this increased risk would have to be balanced with the potential life-saving benefits. This was in agreement with Pezy et al. ${ }^{16}$ who also found overlapping confidence intervals. Thus, a standardized length cannot currently be recommended for Zone 3. In the case of uncontrollable pelvic hemorrhage, one of the possible indications for 
occlusion of Zone 3, in which REBOA might be lifesaving, prehospital occlusion in Zone 1 could be a possible solution, until radiographic guidance is possible.

Prehospital or bedside REBOA might also help a large group of patients with non-hypovolemic and nontraumatic cardiac arrest as a mean to increase central perfusion. Even though standard cardiopulmonary resuscitation may sustain a cardiac output equal to the one seen in heart failure ${ }^{21}$, this is insufficient to obtain or retain a shockable rhythm for many patients ${ }^{22}$.

Patients surviving cardiac arrest have a high risk of brain injury ${ }^{14,23}$. REBOA may redirect the blood flow to the heart and brain and thereby increase both post-cardiac arrest survival and neurological outcome. To expand the use of REBOA, not only a standardized length, but experienced providers are needed to ensure safe and fast insertion of a REBOA catheter.

The finding that safe REBOA balloon placement in Zone 1 is limited to patients resembling those studied in this and previous cohorts ${ }^{16,19}$. Pezy et al. ${ }^{16}$ also found an association with ethnicity, which further underlines the necessity for caution in extrapolation. Finally, measuring the length from baseline to an anatomical landmark with CT images does not consider the actual intra-arterial behavior of a REBOA catheter; thus, the actual intraarterial length of a catheter might deviate from an externally measured length. The measured length is estimated by a line through the center of the lumen following the course of the aorta. A catheter might not follow this exact route, especially in patients with a tortuous aorta ${ }^{24}$.

Notwithstanding these limitations, the identification of a safe interval for REBOA placement is a first step to implement safe prehospital or bedside REBOA.

\section{Conclusion}

Placement of REBOA into Zone 1 using a standardized insertion length may be possible and safe in a population with demographics resembling the present study. Reconciling the present results with previous studies, we recommend targeting the lower part of Zone 1 to minimize the risk of incorrect placement.

Finally, we could not identify a similar safety interval for placement of the REBOA in Zone 3 due to overlapping intervals for the upper and lower anatomical borders of this zone.

\section{Data availability}

The datasets generated and analysed during the current study are not publicly available due to Danish Law but are available from the corresponding author on reasonable request and only after signing a Data Processing Agreement.

Received: 6 January 2020; Accepted: 28 July 2020

Published online: 07 August 2020

\section{References}

1. Morrison, J. J. et al. A systematic review of the use of resuscitative endovascular balloon occlusion of the aorta in the management of hemorrhagic shock. J. Trauma Acute Care Surg. 80, 324-334. https://doi.org/10.1097/TA.0000000000000913 (2016).

2. van Oostendorp, S. E., Tan, E. C. \& Geeraedts, L. M. Prehospital control of life-threatening truncal and junctional haemorrhage is the ultimate challenge in optimizing trauma care; a review of treatment options and their applicability in the civilian trauma setting. Scand. J. Trauma Resusc. Emerg. Med. 24, 1-13. https://doi.org/10.1186/s13049-016-0301-9 (2016).

3. Sesma, J. et al. Effect of intra-aortic occlusion balloon in external thoracic compressions during CPR in pigs. Am. J. Emerg. Med. 20, 453-462. https://doi.org/10.1053/ajem.2002.32627 (2002).

4. Markov, N. P. et al. Physiologic tolerance of descending thoracic aortic balloon occlusion in a swine model of hemorrhagic shock. Surgery (United States) 153, 848-856. https://doi.org/10.1016/j.surg.2012.12.001 (2013).

5. Avaro, J. P. et al. Forty-minute endovascular aortic occlusion increases survival in an experimental model of uncontrolled hemorrhagic shock caused by abdominal trauma. J. Trauma Inj. Infect. Crit. Care 71, 720-725. https://doi.org/10.1097/TA.0b013e3182 21a94a (2011).

6. Ribeiro Junior, M. A. F. et al. The complications associated with Resuscitative Endovascular Balloon Occlusion of the Aorta (REBOA). World J. Emerg. Surg. 13, 20. https://doi.org/10.1186/s13017-018-0181-6 (2018).

7. Stannard, A., Eliason, J. L. \& Rasmussen, T. E. Resuscitative endovascular balloon occlusion of the aorta (REBOA) as an adjunct for hemorrhagic shock. J. Trauma Inj. Infect. Crit. Care 71, 1869-1872. https://doi.org/10.1097/TA.0b013e31823fe90c (2011).

8. Ordoñez, C. A. et al. Current use of resuscitative endovascular balloon occlusion of the aorta (REBOA) in trauma. Colomb. J. Anesthesiol. 45, 30-38. https://doi.org/10.1016/j.rcae.2017.09.007 (2017).

9. Martinelli, T. et al. Intra-aortic balloon occlusion to salvage patients with life-threatening hemorrhagic shocks from pelvic fractures. J. Trauma Inj. Infect. Crit. Care 68, 942-948. https://doi.org/10.1097/TA.0b013e3181c40579 (2010).

10. Irahara, T. et al. Retrospective study of the effectiveness of intra-aortic balloon occlusion (IABO) for traumatic haemorrhagic shock. World J. Emerg. Surg. 10, 1. https://doi.org/10.1186/1749-7922-10-1 (2015).

11. Sadek, S. et al. Resuscitative endovascular balloon occlusion of the aorta (REBOA) in the pre-hospital setting: an additional resuscitation option for uncontrolled catastrophic haemorrhage. Resuscitation 107, 135-138. https://doi.org/10.1016/j.resuscitat ion.2016.06.029 (2016).

12. Wasicek, P. J. et al. Successful and unsuccessful blind placement of resuscitative endovascular balloon occlusion of the aorta (REBOA) catheters through damaged arteries: a report of three cases. J. Endovasc. Resusc. Trauma Manag. 2, 17-22. https://doi. org/10.26676/jevtm.v2i1.43 (2018).

13. Scott, D. J. et al. A novel fluoroscopy-free, resuscitative endovascular aortic balloon occlusion system in a model of hemorrhagic shock. J. Trauma Acute Care Surg. 75, 122-128. https://doi.org/10.1097/TA.0b013e3182946746 (2013).

14. Morrison, J. J. et al. Prospective evaluation of the correlation between torso height and aortic anatomy in respect of a fluoroscopy free aortic balloon occlusion system. Surgery (United States) 155, 1044-1051. https://doi.org/10.1016/j.surg.2013.12.036 (2014).

15. MacTaggart, J. N. et al. Morphometric roadmaps to improve accurate device delivery for fluoroscopy-free resuscitative endovascular balloon occlusion of the aorta. J. Trauma Acute Care Surg. 80, 941-946. https://doi.org/10.1097/TA.0000000000001043 (2016).

16. Pezy, P. et al. Fixed-distance model for balloon placement during fluoroscopy-free resuscitative endovascular balloon occlusion of the aorta in a civilian population. JAMA Surg. 152, 351-358. https://doi.org/10.1001/jamasurg.2016.4757 (2017).

17. Eliason, J. L. et al. CT Correlation of skeletal landmarks and vascular anatomy in civilian adult trauma patients. J. Trauma Acute Care Surg. 87, 1. https://doi.org/10.1097/ta.0000000000002247 (2019). 
18. Team, R. C. R: A Language and Environment for Statistical Computing (R Found. Stat. Comput., Vienna, 2020).

19. Okada, Y., Narumiya, H., Ishi, W. \& Iiduka, R. Anatomical landmarks for safely implementing resuscitative balloon occlusion of the aorta (REBOA) in zone 1 without fluoroscopy. Scand. J. Trauma. Resusc. Emerg. Med. 25, 1-5. https://doi.org/10.1186/s1304 9-017-0411-z (2017).

20. Linnebur, M. et al. Emergent non-image-guided resuscitative endovascular balloon occlusion of the aorta (REBOA) catheter placement: a cadaver-based study. J. Trauma Acute Care Surg. 81, 453-457. https://doi.org/10.1097/TA.0000000000001106 (2016).

21. Fodden, D. I., Crosby, A. C. \& Channer, K. S. Doppler measurement of cardiac output during cardiopulmonary resuscitation. J. Accid. Emerg. Med. 13, 379-382 (1996).

22. Patil, K. D., Halperin, H. R. \& Becker, L. B. Cardiac arrest: resuscitation and reperfusion. Circ. Res. 116, 2041-2049. https://doi. org/10.1161/CIRCRESAHA.116.304495 (2015).

23. Girotra, S., Chan, P. S. \& Bradley, S. M. Post-resuscitation care following out-of-hospital and in-hospital cardiac arrest. Heart 101, 1943-1949. https://doi.org/10.1136/heartjnl-2015-307450 (2015).

24. Liu, S. et al. Transcatheter aortic valve implantation in an extremely tortuous S-shaped aorta. Case Rep. Cardiol. 2017, 2936513. https://doi.org/10.1155/2017/2936513 (2017).

\section{Acknowledgements}

We would like to acknowledge Pelle Baggesgaard Petersen, MD, for sharing his pearls of wisdom to help design this article. The study was funded by Neurescue Aps, who did not have any influence on the manuscript nor the collection of data.

\section{Author contributions}

L.S. and M.H.O. conceived and designed the study. T.T. extracted the data and M.H.O. analysed the data. M.H.O. interpreted the results and prepared the figures. M.H.O. and K.M. drafted the manuscript, and L.S. and T.T. revised the manuscript critically. All authors have seen and approved the final manuscript.

\section{Competing interests}

Dr Olsen has received a grant from Neurescue (a company manufacturing REBOA catheters) in conjunction with writing this manuscript. An agreement was signed, whereby Neurescue had no influence on the writing and wording of the manuscript but retained the right to review the manuscript for proprietary information prior to submission. The rest of the authors (T.T., L.S., K.M.) certify that they have no affiliations with or involvement in any organization or entity with any financial interest (such as honoraria; educational grants; participation in speakers' bureaus; membership, employment, consultancies, stock ownership, or other equity interest; and expert testimony or patent-licensing arrangements), or non-financial interest (such as personal or professional relationships, affiliations, knowledge or beliefs) in the subject matter or materials discussed in this manuscript.

\section{Additional information}

Supplementary information is available for this paper at https://doi.org/10.1038/s41598-020-70364-9.

Correspondence and requests for materials should be addressed to M.H.O.

Reprints and permissions information is available at www.nature.com/reprints.

Publisher's note Springer Nature remains neutral with regard to jurisdictional claims in published maps and institutional affiliations.

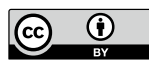

Open Access This article is licensed under a Creative Commons Attribution 4.0 International License, which permits use, sharing, adaptation, distribution and reproduction in any medium or format, as long as you give appropriate credit to the original author(s) and the source, provide a link to the Creative Commons license, and indicate if changes were made. The images or other third party material in this article are included in the article's Creative Commons license, unless indicated otherwise in a credit line to the material. If material is not included in the article's Creative Commons license and your intended use is not permitted by statutory regulation or exceeds the permitted use, you will need to obtain permission directly from the copyright holder. To view a copy of this license, visit http://creativecommons.org/licenses/by/4.0/.

(C) The Author(s) 2020 